\title{
Two-Dimensional Optical Signal Processing
}

\author{
Theo Kooij \\ Defense Advanced Research Projects Agency \\ 1400 Wilson Boulevard \\ Arlington, Virginia 22209 \\ Jacques E. Ludman \\ Rome Air Development Center \\ Hanscom Air Force Base, Massachusetts 01731 \\ P. Denzil Stilwell, Jr. \\ Naval Research Laboratory \\ Code 7924 \\ Washington, D.C. 20375
}

When some optical processing systems firms proposed to the Defense Advanced Research Project Agency (DARPA) and the U.S. Navy some years ago that they could beat the ILLIAC-IV-that venerable supercomputer, which until recently was the world's largest-by at least a factor of 100 , it sounded too good to be true. But they were right, and they did not even have to try hard. The problem was a two-dimensional (2-D) processing task of generating ambiguity surfaces to test whether two received signals came from a common origin, with unknown time and Doppler shifts. The ILLIAC, going all out as an in-line processor for the Acoustic Research Center near San Francisco, California, could just make a handful of such surfaces per second; the optical processors made hundreds, literally sucking their digital inputs dry.

Where had this optical signal processing wonder child been? And where else could its power be applied? Surely there would be other two-dimensional processing problems begging for equally simple solutions. To explore this notion, a symposium was held at the Naval Research Laboratory in Washington, D.C., in February 1981, entitled "Two-Dimensional Signal Processing." Optical implementations were sought for a variety of signal processors operating on more than one parameter: time-Doppler, time and spatial frequency, frequency-azimuth, phase planes, etc. The papers in this special issue cover some of the topics that were presented at that symposium.

The first paper in this issue ( $\mathrm{Szu}$ ) is about the 2-D processing of acoustic information, and the next two (Casasent and Carlotto; Psaltis and Wagner) are about radar processing, one for beam-forming purposes, the other for signal processing. The phase retrieval paper (Gonsalves) is generic to all 2-D processing. A presentation at the symposium on electronic systolic processing prompted a collaboration between two of the special editors (Ludman, Stilwell) and the journal editor (Caulfield) which resulted in the paper on optical systolic processing. From this sample of applications and prospects, it is clear that 2-D processing is yet in its infancy, and as it matures, the uses and applications will proliferate.

One of us (J. Ludman), by virtue of proximity to the journal editor, has learned a great deal more about editing than anticipated. For example, at a certain point in time, having equations numbered correctly and having the figures right side up and connected to the proper paper become as important as their correctness or information content. 